\title{
Chronic dietary exposure to pesticide residues in the United States
}

\author{
Carl K Winter
}

\begin{abstract}
Background: Discussions as to the extent of pesticide residue contamination in the food supply often rely on results of government residue monitoring programs focusing primarily upon the percentages of samples containing pesticide residues and the number of violative residues identified. Such an approach does not adequately convey the likelihood of pesticide residues posing consumer risks since residue regulatory limits are not safety standards and violative pesticide residues rarely constitute residues of health concern. It is more appropriate to develop estimates of actual dietary exposure to pesticides and to compare such estimates to established toxicological criteria such as the Chronic Reference Dose (RfD). The U.S. Food and Drug Administration's (FDA) Total Diet Study (TDS) previously provided such information but last published its findings in 1995 to estimate dietary exposure to pesticides detected between 1986 and 1991. This paper provides updated estimates of dietary exposure to pesticides in the United States using the most recent TDS findings on pesticide residues.
\end{abstract}

Results: A total of 77 specific pesticides were detected from market basket samples of 2240 TDS food items analyzed by FDA in 2004 and 2005. All estimated exposures to the 77 pesticides for the General US population were well below chronic RfD levels. Only 3 of the 77 pesticides showed exposures greater than $1 \%$ of chronic RfDs, while 14 showed exposures between 0.1 and $1 \%$ of chronic RfDs and 19 had exposures between 0.01 and $0.1 \%$ of chronic RfDs. The remaining 41 pesticides had exposures below $0.01 \%$ of chronic RfDs.

Compared with 1986-1991 findings, dietary exposure to six environmentally persistent chlorinated hydrocarbon insecticides were reduced by factors of 47 to $96 \%$ in 2004-2005. Exposures to 15 different population subgroups were estimated and indicated that children, particularly two year-olds, frequently receive higher exposures to pesticide residues in their diets than do adults.

Conclusions: Chronic dietary exposure to pesticides in the diet, according to results of the FDA's 2004-2005 TDS, continue to be at levels far below those of health concern. Consumers should be encouraged to eat fruits, vegetables, and grains and should not fear the low levels of pesticide residues found in such foods.

Keywords: Pesticides; Food safety; Risk assessment; Exposure assessment; Total diet study; Reference dose

\section{Background}

Residues of pesticide chemicals are frequently detected on the edible portions of foods and consumers are routinely exposed to pesticide residues in their diets. The extent of pesticide contamination in the food supply is often discussed in terms of the results of regulatory monitoring conducted by bodies such as the U.S. Food and Drug Administration (FDA) and the European Food Safety Authority (EFSA). In 2012, FDA monitoring results of 1,158 domestic samples showed that $57 \%$ had

Correspondence: ckwinter@ucdavis.edu

Department of Food Science and Technology, University of California, Davis, CA, USA no detectable residues while $2.8 \%$ contained violative pesticide residues (FDA 2015). Among the 4,365 imported food samples analyzed, $66 \%$ had no detectable residues while violations were detected on $11.1 \%$ of the samples. EFSA reported on the analysis of 80,967 food samples collected in 2013 and concluded that $54.6 \%$ of the samples had no detectable residues while $2.6 \%$ of the samples resulted in violations (EFSA 2015).

Such findings may be confusing to consumers since the majority of samples contained no detectable residues of pesticides although pesticide residue violations also frequently occurred. Unfortunately, while counterintuitive, the recitation of findings from regulatory monitoring programs

\section{Springer}


is of little value in terms of assessing the potential health risks posed by pesticide residues in foods. This is primarily due to the fact that the allowable levels are not indicators of safety but rather reflect enforcement tools to assess whether Good Agricultural Practices have been followed (Winter, 1992). As such, residue violations often indicate breaches of Good Agricultural Practices but only in very rare circumstances represent cases of health concern. To clarify the differences between allowable pesticide residue levels and levels of health significance, a companion system to establish levels of safety concerns for specific pesticides on specific food items has been proposed (Winter and Jara, 2015).

The potential health risks posed by pesticide residues in foods can best be assessed by developing estimates of dietary exposure to pesticides and comparing exposure estimates to toxicological indicators of health concern such as the Chronic Reference Dose (RfD) or the analogous Acceptable Daily Intake (ADI). An accurate estimation of dietary pesticide exposure requires data on specific levels of pesticide residues detected (not just whether the residues were legal or violative) as well as estimations of consumption amounts of all foods for which residues are detected.

The FDA has conducted the Total Diet Study (TDS) annually since 1961 (Gunderson 1995). The TDS uses a market basket approach in which FDA inspectors purchase market baskets of 280 food items from retail locations in various regions of the country. The food items are prepared for consumption and then analyzed for potential food contaminants such as radionuclides and pesticide residues as well as for metals and selected nutrients. Food consumption estimates for the TDS diets are derived from national food consumption databases. By multiplying the levels of pesticide residues found on the food items by food consumption estimates, it is possible to obtain an estimate of chronic dietary exposure to pesticide residues in foods.

Historically, FDA released its estimates of chronic dietary exposure to pesticides obtained from TDS findings and compared exposure estimates to ADI levels. Unfortunately, the last public report of such findings was made in 1995 and estimated dietary exposure to pesticides from TDS market baskets collected between 1986 and 1991 (Gunderson 1995). Thus, while FDA continues to analyze TDS samples for pesticide residues, it has not made its findings of TDS consumer exposure to pesticides available for two decades.

At the same time, FDA does release the analytical data resulting from subsequent TDS market baskets as well as food consumption estimates for each of the TDS foods. It is therefore possible to estimate dietary exposure to pesticides from TDS data and to compare exposure levels to chronic RfD or ADI levels.
This paper provides estimates of dietary exposure to pesticides based upon the most recent two years (2004 and 2005) for which TDS pesticide residue data are available. Exposure estimates are compared with chronic RfD values to determine the potential risks associated with the exposures. In addition, comparisons between exposures from the 2004-2005 and the 1986-1991 market baskets are made to identify longer-term trends in dietary exposure to pesticides.

\section{Methods}

Pesticide residue levels on the 280 TDS foods were obtained from FDA's summary of pesticide residue findings for Market Baskets 2004-1 through 2005-4 (FDA 2014). While analytical methods were capable of detection of more than 300 pesticides and/or pesticide metabolites or breakdown products, residues were detected for 77 specific pesticides. According to FDA guidelines, a value of 0 was assigned for residues below the analytical method's limit of detection while trace findings were reported at the measured value.

Food consumption estimates for each of the 280 TDS foods were acquired by downloading FDA's "TDS Diets, Version 3" (FDA 2013). Food consumption estimates were derived from the results of the United States Department of Agriculture's 1994-96 and 1998 Continuing Survey of Food Intakes by Individuals. Consumption estimates were expressed in terms of grams of food per day and were separated into 15 population subgroups: Total US population, infants $6-11$ months, children 2 years, children 6 years, children $6-10$ years, males (14-16 years, $25-30$ years, $40-45$ years, $60-65$ years, 70 years) and females (14-16 years, $25-30$ years, $40-45$ years, $60-65$ years, 70 years).

To estimate dietary exposure to specific pesticides for each of the population subgroups, the mean residue level (expressed in $\mathrm{mg} / \mathrm{kg}$ ) found on a specific TDS food was multiplied by the food consumption estimate to yield an estimate of the daily exposure to the pesticide on that food. If, as was common, a pesticide was detected on more than one TDS food, contributing exposures from each food were combined to yield a daily exposure estimate to the pesticide.

To allow direct comparisons between chronic RfD values and exposure estimates, daily pesticide exposure levels were divided by body weight levels estimated for each population subgroup (EPA 2011). Final chronic dietary exposures to specific pesticides for each population subgroup were then expressed in terms of nanograms pesticide/kg body weight/day (ng/kg/day).

The methods used to estimate chronic dietary exposure to pesticides represent a simplistic deterministic approach in which residues are considered to be present at mean levels and food consumption estimates are chosen 
that represent the $50^{\text {th }}$ percentile for each of the 280 foods considered. As such, the approach attempts to most accurately characterize typical chronic dietary exposure to pesticides. The use of higher percentiles of residue level or food consumption estimates would serve to artificially inflate exposure estimates. Deterministic methods using higher percentiles of residue levels and/ or food consumption estimates are useful, and necessary, in assessing acute exposure to pesticides in foods but their use in a chronic exposure assessment is not warranted.

Chronic RfD values for specific pesticides were primarily obtained from the Integrated Risk Information System (IRIS) used by the United States Environmental Protection Agency (EPA). The chronic RfD represents an estimate of the amount of a chemical an individual could be exposed to daily throughout the individual's lifetime that is likely to be without an appreciable risk of harm (Winter and Francis 1997). In some cases, chronic RfD values were not available through IRIS but could be identified through documents discussing EPA Pesticide Reregistration Eligibility Decisions for specific pesticides. Chronic RfD values were not obtained for the pesticides azinphos-methyl, chlorpyrifos-methyl, dicloran, dicofol, fenhexamid, omethoate, phenylphenol-ortho, quinoxyfen, tecnazene, thiabendazole, triadimefon, and trifloxystrobin; ADI values from the literature were chosen as chronic RfD surrogates for each of these pesticides.

\section{Results and discussion}

The FDA analyzed a total of 2240 food items (8 market baskets x 280 items per market basket) in its 2004 and 2005 TDS for residues of more than 300 pesticides and/ or metabolites and breakdown products. Residues of 77 pesticides were detected.

The estimated chronic dietary exposures to the 77 pesticides for the General US population are compared with chronic RfD values in Table 1. Exposure levels to all 77 pesticides were below chronic RfD values. Exposures to 3 pesticides exceeded $1 \%$ of the chronic RfD values while exposures between 0.1 and $1 \%$ of chronic RfD values were noted for 14 pesticides. Another 19 pesticides demonstrated exposures between 0.01 and $0.1 \%$ of the chronic RfD values, while exposures for the other 41 pesticides were below $0.01 \%$ of the chronic RfD. The median exposure relative to the chronic RfD for the 77 pesticides was for the fungicide captan, with an exposure of $0.0074 \%$ of the chronic RfD.

The determination of chronic RfD values requires assessment of the No Observed Adverse Effect Level (NOAEL), which represents the highest continuous dose given to the most sensitive animal species that does not demonstrate any noticeable toxicity (Winter and Francis 1997). The NOAEL levels are typically reduced using uncertainty factors (100 or, in some cases 1000$)$ to produce the chronic
Table 1 Comparison of General US dietary exposure to pesticides with Chronic Reference Doses (RfDs)

\begin{tabular}{|c|c|c|c|}
\hline Pesticide & $\begin{array}{l}\text { General US } \\
\text { Exposure } \\
(\mathrm{ng} / \mathrm{kg} / \text { day) }\end{array}$ & $\begin{array}{l}\text { Chronic RfD } \\
\text { (ng/kg/day) }\end{array}$ & $\% \mathrm{RfD}$ \\
\hline Acephate & 18.4281 & 4000 & 0.4607 \\
\hline Atrazine & 0.0072 & 35000 & $<0.0001$ \\
\hline Azinphos-methyl & 11.1229 & 5000 & 0.2225 \\
\hline Azoxystrobin & 1.1496 & 180000 & 0.0006 \\
\hline Benomyl & 9.2942 & 50000 & 0.0186 \\
\hline Bifenthrin & 0.9997 & 15000 & 0.0067 \\
\hline Biphenyl & 0.3963 & 500000 & 0.0001 \\
\hline Captan & 9.6072 & 130000 & 0.0074 \\
\hline Carbaryl & 10.2073 & 100000 & 0.0102 \\
\hline Carbofuran & 0.3657 & 5000 & 0.0073 \\
\hline Chlordane (total) & 0.0992 & 500 & 0.0198 \\
\hline Chlordane, cis & 0.0479 & & \\
\hline Chlordane, trans & 0.0125 & & \\
\hline Nonachlor, trans & 0.0367 & & \\
\hline Octachlor epoxide & 0.0020 & & \\
\hline Chloropropham & 287.7204 & 200000 & 0.1439 \\
\hline Chlorothalonil & 0.1205 & 15000 & 0.0008 \\
\hline Chlorpyrifos & 2.3760 & 3000 & 0.0792 \\
\hline Chlorpyrifos-methyl & 12.7964 & 10000 & 0.1280 \\
\hline Clopyralid & 0.8296 & 150000 & 0.0006 \\
\hline Coumaphos & 0.0068 & 700 & 0.0010 \\
\hline Cyfluthrin & 0.2220 & 24000 & 0.0009 \\
\hline Cypermethrin & 8.9699 & 10000 & 0.0897 \\
\hline Cyprodinil & 12.9971 & 37500 & 0.0347 \\
\hline $2,4-D$ & 0.6738 & 10000 & 0.0067 \\
\hline DCPA & 0.2398 & 10000 & 0.0024 \\
\hline DDT, total & 6.6482 & 500 & 1.3296 \\
\hline$D D E, p, p^{\prime}$ & 5.9085 & & \\
\hline DDT, o, p' & 0.0120 & & \\
\hline DDT, $p, p^{\prime}$ & 0.1471 & & \\
\hline $\mathrm{TDE}, \mathrm{o}, \mathrm{p}^{\prime}$ & 0.0258 & & \\
\hline TDE, $p, p^{\prime}$ & 0.5547 & & \\
\hline DEF & 0.1086 & 100 & 0.1086 \\
\hline Diazinon & 0.2394 & 200 & 0.1197 \\
\hline Dicamba & 0.7488 & 30000 & 0.0025 \\
\hline Dichloran, total & 2.9128 & 2500 & 0.1165 \\
\hline Dichloran & 2.8691 & & \\
\hline 2,4-Dichloro-6-nitrobenzeneamine & 0.0437 & & \\
\hline Dicofol, total & 0.3547 & 200000 & 0.0002 \\
\hline Dicofol, o, p' & 0.0345 & & \\
\hline Dicofol, p, p' & 0.3201 & & \\
\hline
\end{tabular}


Table 1 Comparison of General US dietary exposure to pesticides with Chronic Reference Doses (RfDs) (Continued)

\begin{tabular}{|c|c|c|c|}
\hline Dieldrin & 0.9841 & 50 & 1.9683 \\
\hline Dimethoate & 1.5595 & 200 & 0.7797 \\
\hline Diphenylamine & 79.6903 & 25000 & 0.3188 \\
\hline Endosulfan, total & 9.3737 & 6000 & 0.1562 \\
\hline Endosulfan I & 1.6268 & & \\
\hline Endosulfan ॥ & 2.1831 & & \\
\hline Endosulfan sulfate & 5.5638 & & \\
\hline Endrin & 0.0228 & 300 & 0.0076 \\
\hline Esfenvalerate & 0.0281 & 1800 & 0.0016 \\
\hline Ethion, total & 0.4912 & 500 & 0.0982 \\
\hline Ethion & 0.4869 & & \\
\hline Ethion, oxygen analog & 0.0043 & & \\
\hline Ethoxyquin & 0.5940 & 20000 & 0.0030 \\
\hline Famoxadone & 0.1185 & 1400 & 0.0085 \\
\hline Fenarimol & 0.0196 & 65000 & $<0.0001$ \\
\hline Fenhexamid & 0.9919 & 200000 & 0.0005 \\
\hline Fenitrothion & 0.0132 & 1500 & 0.0009 \\
\hline Fenoxaprop-ethyl & 0.0002 & 15000 & $<0.0001$ \\
\hline Fenpropathrin & 3.3847 & 25000 & 0.0135 \\
\hline Fenvalerate & 0.3790 & 25000 & 0.0015 \\
\hline Fludioxonil & 21.1253 & 30000 & 0.0704 \\
\hline Heptachlor & 0.0593 & 13 & 0.4563 \\
\hline Imazalil & 0.5742 & 13000 & 0.0044 \\
\hline Iprodione, total & 6.4931 & 40000 & 0.0162 \\
\hline Iprodione & 6.1491 & & \\
\hline Iprodione metabolite & 0.3441 & & \\
\hline Lambda-cyhalothrin & 0.6892 & 5000 & 0.0138 \\
\hline Lindane & 0.0693 & 300 & 0.0231 \\
\hline Linuron & 0.2379 & 2000 & 0.0119 \\
\hline Malathion & 29.6982 & 20000 & 0.1485 \\
\hline Metalaxyl & 1.0858 & 60000 & 0.0018 \\
\hline Methamidophos & 7.9984 & 50 & 15.9968 \\
\hline Methidathion & 0.9529 & 1000 & 0.0953 \\
\hline Methomyl & 1.9347 & 25000 & 0.0077 \\
\hline Methoxychlor, total & 0.0428 & 5000 & 0.0009 \\
\hline Methoxychlor, o, p' & 0.0173 & & \\
\hline Methoxychlor, p, p' & 0.0255 & & \\
\hline Mirex & 0.0020 & 200 & 0.0010 \\
\hline Omethoate & 0.8798 & 300 & 0.2933 \\
\hline Oxamyl & 0.0466 & 25000 & 0.0002 \\
\hline Permethrin, total & 73.4122 & 50000 & 0.1468 \\
\hline Permethrin, cis & 35.4246 & & \\
\hline Permethrin, trans & 37.9876 & & \\
\hline Phenylphenol, ortho & 11.3419 & 20000 & 0.0567 \\
\hline
\end{tabular}

Table 1 Comparison of General US dietary exposure to pesticides with Chronic Reference Doses (RfDs) (Continued)

\begin{tabular}{llll}
\hline Phosalone & 0.0491 & 2000 & 0.0025 \\
Phosmet & 4.7271 & 20000 & 0.0236 \\
Piperonyl butoxide & 0.0029 & 6300000 & $<0.0001$ \\
Pirimiphos-methyl & 6.3524 & 10000 & 0.0635 \\
Procymidone & 0.0247 & 35000 & 0.0001 \\
Propargite & 11.6608 & 20000 & 0.0583 \\
Propiconazole & 0.0002 & 13000 & $<0.0001$ \\
Quinclorac & 1.0475 & 380000 & 0.0003 \\
Quinoxyfen & 0.2185 & 200000 & 0.0001 \\
Quintozene & 0.0743 & 3000 & 0.0025 \\
Tecnazene & 0.0008 & 20000 & $<0.0001$ \\
Thiabendazole & 77.7198 & 100000 & 0.0777 \\
Triadimefon & 0.0033 & 10000 & $<0.0001$ \\
Triclopyr & 0.0398 & 50000 & 0.0001 \\
Trifloxystrobin & 0.3277 & 100000 & 0.0003 \\
Vinclozolin & 0.8230 & 25000 & 0.0033 \\
\hline
\end{tabular}

RfD level. In the case of dietary exposure to a pesticide that represents $0.01 \%$ of the chronic RfD, the exposure level (assuming the use of a 100 fold uncertainty factor in deriving the RfD) is one million times lower than levels that do not cause any noticeable effects in animals dosed continuously with the pesticide throughout their lifetimes. Thus, findings from this study reinforce the notion that typical consumer exposure to pesticide residues is at levels far below those of health concern.

The highest exposure of a pesticide relative to the chronic RfD was for methamidophos, which showed an exposure to the General US population of $16 \%$ of the chronic RfD. Methamidophos is an organophosphate insecticide that shares a common toxicological mechanism with several other common insecticides. The estimated exposure to methamidophos for the General US population, $8.0 \mathrm{ng} / \mathrm{kg} /$ day, is actually lower than estimated exposures to other organophosphate insecticides such as

Table 2 Comparison of chlorinated hydrocarbon insecticide dietary exposure for two year-olds, 2004-05 vs. 1986-91 ${ }^{\text {a }}$

\begin{tabular}{lccc}
\hline Pesticide & $\begin{array}{l}2004-05 \\
(\mathrm{ng} / \mathrm{kg} / \text { day })\end{array}$ & $\begin{array}{l}1986-91 \\
(\mathrm{ng} / \mathrm{kg} / \text { day })\end{array}$ & $\begin{array}{c}\text { Percentage } \\
\text { reduction }\end{array}$ \\
\hline DDT & 23.18 & 43.8 & 47 \\
Dicofol & 1.52 & 35.5 & 96 \\
Dieldrin & 2.7 & 7.2 & 63 \\
Heptachlor & 0.15 & 2.5 & 94 \\
Lindane & 0.26 & 3.2 & 92 \\
Methoxychlor & 0.1 & 0.9 & 89 \\
\hline a 1986-91 data obtained from Gunderson (1995) & &
\end{tabular}


Table 3 Dietary pesticide exposure estimates for specific population subgroups (ng/kg/day) ${ }^{a}$

\begin{tabular}{|c|c|c|c|c|c|c|}
\hline Pesticide & US General & 6-11 month & $M / F 2$ years & M/F 6 years & M/F 10 year & F 14-16 \\
\hline Acephate & 18.4281 & 17.2302 & 42.6352 & 29.8366 & 17.0595 & 8.5112 \\
\hline Atrazine & 0.0072 & 0.0103 & 0.0203 & 0.0096 & 0.0052 & 0.0047 \\
\hline Azinphos-methyl & 11.1229 & 32.5191 & 82.2131 & 47.7940 & 26.9670 & 9.1684 \\
\hline Azoxystrobin & 1.1496 & 0.8092 & 4.6546 & 2.2937 & 2.1211 & 1.1600 \\
\hline Benomyl & 9.2942 & 57.5515 & 70.8385 & 34.5814 & 17.4725 & 7.3732 \\
\hline Bifenthrin & 0.9997 & 2.8008 & 2.1272 & 1.6090 & 0.6109 & 0.3124 \\
\hline Biphenyl & 0.3963 & 0.8070 & 1.4732 & 1.1553 & 0.6452 & 0.4489 \\
\hline Captan & 9.6072 & 7.0953 & 72.0200 & 34.5332 & 21.3902 & 9.9809 \\
\hline Carbaryl & 10.2073 & 64.3193 & 83.9221 & 41.4619 & 18.4712 & 11.6797 \\
\hline Carbofuran & 0.3657 & 0.2989 & 1.2256 & 1.3252 & 0.4160 & 0.1333 \\
\hline Chlordane (total) & 0.0992 & 0.0698 & 0.1761 & 0.1504 & 0.1270 & 0.0739 \\
\hline Chlordane, cis & 0.0479 & 0.0220 & 0.0914 & 0.0790 & 0.0598 & 0.0378 \\
\hline Chlordane, trans & 0.0125 & 0.0146 & 0.0234 & 0.0172 & 0.0198 & 0.0086 \\
\hline Nonachlor, trans & 0.0367 & 0.0296 & 0.0585 & 0.0511 & 0.0461 & 0.0267 \\
\hline Octachlor epoxide & 0.0020 & 0.0036 & 0.0028 & 0.0032 & 0.0012 & 0.0007 \\
\hline Chloropropham & 287.7204 & 242.2743 & 981.7293 & 785.8608 & 528.4143 & 351.6736 \\
\hline Chlorothalonil & 0.1205 & 0.0000 & 0.2162 & 0.1754 & 0.1824 & 0.0259 \\
\hline Chlorpyrifos & 2.3760 & 2.5690 & 9.8882 & 5.5129 & 3.8547 & 2.2513 \\
\hline Chlorpyrifos-methyl & 12.7964 & 12.4004 & 40.6889 & 37.3143 & 23.2516 & 13.4883 \\
\hline Clopyralid & 0.8296 & 2.3563 & 5.2877 & 3.2069 & 1.8738 & 0.6758 \\
\hline Coumaphos & 0.0068 & 0.0025 & 0.0218 & 0.0109 & 0.0014 & 0.0028 \\
\hline Cyfluthrin & 0.2220 & 0.0079 & 0.1208 & 0.1013 & 0.1664 & 0.0467 \\
\hline Cypermethrin & 8.9699 & 8.1437 & 17.1238 & 10.4601 & 3.8659 & 6.0168 \\
\hline Cyprodinil & 12.9971 & 11.9101 & 119.2924 & 47.1034 & 31.9188 & 14.6596 \\
\hline $2,4-D$ & 0.6738 & 0.5266 & 2.1795 & 1.8683 & 1.1665 & 0.5975 \\
\hline DCPA & 0.2398 & 0.1450 & 0.5591 & 0.3654 & 0.2523 & 0.1717 \\
\hline DDT, total & 6.6482 & 8.0532 & 23.1850 & 18.0708 & 11.8551 & 6.3338 \\
\hline DDE, p, $p^{\prime}$ & 5.9085 & 7.3662 & 21.6413 & 16.9921 & 11.1399 & 5.7689 \\
\hline DDT, o, p' & 0.0120 & 0.0062 & 0.0156 & 0.0098 & 0.0070 & 0.0100 \\
\hline DDT, p, p' & 0.1471 & 0.3271 & 0.6385 & 0.3535 & 0.2306 & 0.1427 \\
\hline$T D E, o, p^{\prime}$ & 0.0258 & 0.0530 & 0.0241 & 0.0231 & 0.0076 & 0.0122 \\
\hline
\end{tabular}


Table 3 Dietary pesticide exposure estimates for specific population subgroups ( $\mathrm{ng} / \mathrm{kg} / \mathrm{day}$ ) ${ }^{a}$ (Continued)

\begin{tabular}{|c|c|c|c|c|c|c|}
\hline TDE, p, p' & 0.5547 & 0.3008 & 0.8654 & 0.6923 & 0.4700 & 0.4000 \\
\hline DEF & 0.1086 & 0.0153 & 0.4647 & 0.3953 & 0.2353 & 0.1265 \\
\hline Diazinon & 0.2394 & 0.0658 & 0.3905 & 0.3455 & 0.2217 & 0.2152 \\
\hline Dicamba & 0.7488 & 2.2202 & 4.8366 & 3.0227 & 1.8074 & 0.6574 \\
\hline Dichloran, total & 2.9128 & 6.4696 & 5.3965 & 3.9703 & 3.9514 & 1.6418 \\
\hline Dichloran & 2.8691 & 6.4696 & 5.3180 & 3.9066 & 3.8853 & 1.6323 \\
\hline 2,4-Dichloro-6-nitrobenzeneamine & 0.0437 & 0.0000 & 0.0784 & 0.0636 & 0.0662 & 0.0094 \\
\hline Dicofol, total & 0.3547 & 0.4972 & 1.5264 & 0.9061 & 0.6697 & 0.2927 \\
\hline Dicofol, o, p' & 0.0345 & 0.0465 & 0.2390 & 0.1394 & 0.0934 & 0.0248 \\
\hline Dicofol, $p, p^{\prime}$ & 0.3201 & 0.4508 & 1.2874 & 0.7667 & 0.5763 & 0.2679 \\
\hline Dieldrin & 0.9841 & 1.5196 & 2.7181 & 2.1000 & 1.5493 & 0.7663 \\
\hline Dimethoate & 1.5595 & 9.5053 & 6.1889 & 3.5457 & 1.8318 & 1.0376 \\
\hline Diphenylamine & 79.6903 & 98.6515 & 606.5610 & 395.4847 & 180.9575 & 70.1601 \\
\hline Endosulfan, total & 9.3737 & 11.0237 & 21.0158 & 16.0028 & 10.7486 & 6.8948 \\
\hline Endosulfan I & 1.6268 & 1.8331 & 4.4537 & 3.0479 & 2.2759 & 1.4302 \\
\hline Endosulfan II & 2.1831 & 2.4196 & 6.1116 & 4.1611 & 2.9966 & 1.8655 \\
\hline Endosulfan sulfate & 5.5638 & 6.7710 & 10.4504 & 8.7938 & 5.4761 & 3.5992 \\
\hline Endrin & 0.0228 & 0.0627 & 0.0158 & 0.0187 & 0.0025 & 0.0075 \\
\hline Esfenvalerate & 0.0281 & 1.1880 & 0.1006 & 0.0843 & 0.0328 & 0.0416 \\
\hline Ethion, total & 0.4912 & 0.1987 & 1.3184 & 1.3622 & 0.9313 & 0.6354 \\
\hline Ethion & 0.4869 & 0.1979 & 1.3153 & 1.3588 & 0.9288 & 0.6332 \\
\hline Ethion, oxygen analog & 0.0043 & 0.0008 & 0.0031 & 0.0035 & 0.0025 & 0.0022 \\
\hline Ethoxyquin & 0.5940 & 0.0848 & 0.7868 & 1.6565 & 0.8585 & 1.0186 \\
\hline Famoxadone & 0.1185 & 0.0042 & 0.0645 & 0.0541 & 0.0888 & 0.0249 \\
\hline Fenarimol & 0.0196 & 0.0104 & 0.1187 & 0.0392 & 0.0066 & 0.0055 \\
\hline Fenhexamid & 0.9919 & 0.8467 & 5.9062 & 2.5504 & 2.0440 & 1.0660 \\
\hline Fenitrothion & 0.0132 & 0.0118 & 0.0191 & 0.0261 & 0.0146 & 0.0120 \\
\hline Fenoxaprop-ethyl & 0.0002 & 0.1076 & 0.0002 & 0.0000 & 0.0000 & 0.0000 \\
\hline Fenpropathrin & 3.3847 & 15.5940 & 25.9598 & 13.3071 & 7.3449 & 3.1080 \\
\hline Fenvalerate & 0.3790 & 3.0032 & 1.0053 & 0.5161 & 0.2748 & 0.2500 \\
\hline Fludioxonil & 21.1253 & 33.0302 & 66.5408 & 56.8803 & 13.5351 & 26.1458 \\
\hline Heptachlor & 0.0593 & 0.0534 & 0.1478 & 0.1303 & 0.0978 & 0.0489 \\
\hline Imazalil & 0.5742 & 0.1826 & 0.3263 & 0.1806 & 0.1094 & 0.0859 \\
\hline Iprodione, total & 6.4931 & 11.0094 & 26.2975 & 18.0616 & 5.8599 & 7.8378 \\
\hline Iprodione & 6.1491 & 10.3505 & 25.2904 & 17.2475 & 5.6553 & 7.4416 \\
\hline
\end{tabular}


Table 3 Dietary pesticide exposure estimates for specific population subgroups (ng/kg/day) ${ }^{a}$ (Continued)

\begin{tabular}{|c|c|c|c|c|c|c|}
\hline Iprodione metabolite & 0.3441 & 0.6589 & 1.0071 & 0.8142 & 0.2046 & 0.3961 \\
\hline Lambda-cyhalothrin & 0.6892 & 3.5221 & 1.2727 & 0.5294 & 0.2707 & 0.6808 \\
\hline Lindane & 0.0693 & 0.0272 & 0.2586 & 0.2933 & 0.1840 & 0.0942 \\
\hline Linuron & 0.2379 & 0.2362 & 0.5644 & 0.5572 & 0.3341 & 0.1655 \\
\hline Malathion & 29.6982 & 24.4021 & 94.4458 & 89.6674 & 57.0931 & 29.6243 \\
\hline Metalaxyl & 1.0858 & 0.7104 & 9.1932 & 3.6071 & 2.3705 & 1.1077 \\
\hline Methamidophos & 7.9984 & 13.9201 & 15.9612 & 9.7545 & 7.1500 & 4.5228 \\
\hline Methidathion & 0.9529 & 0.7878 & 5.6458 & 3.3187 & 2.2614 & 1.3605 \\
\hline Methomyl & 1.9347 & 1.1424 & 6.5387 & 3.6508 & 3.0433 & 1.7831 \\
\hline Methoxychlor, total & 0.0428 & 0.0244 & 0.1006 & 0.0846 & 0.0622 & 0.0199 \\
\hline Methoxychlor, o, p' & 0.0173 & 0.0000 & 0.0000 & 0.0000 & 0.0000 & 0.0000 \\
\hline Methoxychlor, p, p' & 0.0255 & 0.0244 & 0.1006 & 0.0846 & 0.0622 & 0.0198 \\
\hline Mirex & 0.0020 & 0.0010 & 0.0040 & 0.0024 & 0.0042 & 0.0021 \\
\hline Omethoate & 0.8798 & 3.6634 & 1.7655 & 1.0999 & 0.8271 & 0.5772 \\
\hline Oxamyl & 0.0466 & 0.0024 & 0.0764 & 0.0775 & 0.0538 & 0.0211 \\
\hline Permethrin, total & 73.4122 & 88.3346 & 101.6469 & 80.6081 & 28.9863 & 22.4073 \\
\hline Permethrin, cis & 35.4246 & 42.4138 & 48.9996 & 38.7410 & 14.0356 & 11.0349 \\
\hline Permethrin, trans & 37.9876 & 45.9208 & 52.6472 & 41.8671 & 14.9507 & 11.3723 \\
\hline Phenylphenol, ortho & 11.3419 & 36.9188 & 22.7534 & 17.2762 & 13.4283 & 7.9713 \\
\hline Phosalone & 0.0491 & 0.1417 & 0.0232 & 0.0477 & 0.0617 & 0.0561 \\
\hline Phosmet & 4.7271 & 17.3239 & 19.7964 & 15.2866 & 4.9499 & 5.3718 \\
\hline Piperonyl butoxide & 0.0029 & 1.5171 & 0.0307 & 0.0000 & 0.0000 & 0.0000 \\
\hline Pirimiphos-methyl & 6.3524 & 2.0673 & 19.2953 & 19.6057 & 15.6757 & 4.3406 \\
\hline Procymidone & 0.0247 & 0.0127 & 0.0508 & 0.0672 & 0.0407 & 0.0377 \\
\hline Propargite & 11.6608 & 16.8366 & 55.0796 & 33.4737 & 6.9992 & \\
\hline Propiconazole & 0.0002 & 0.1076 & 0.0002 & 0.0000 & 0.0000 & 0.0000 \\
\hline Quinclorac & 1.0475 & 2.4162 & 3.8001 & 2.5547 & 1.6717 & 0.9916 \\
\hline Quinoxyfen & 0.2185 & 0.1696 & 2.1860 & 0.8194 & 0.5358 & 0.2512 \\
\hline Quintozene & 0.0743 & 0.0952 & 0.2157 & 0.1604 & 0.1027 & 0.0518 \\
\hline Tecnazene & 0.0008 & 0.0003 & 0.0009 & 0.0008 & 0.0010 & 0.0008 \\
\hline Thiabendazole & 77.7198 & 297.9485 & 615.7219 & 346.4239 & 157.2699 & 67.4992 \\
\hline Triadimefon & 0.0033 & 0.0090 & 0.0348 & 0.0024 & 0.0058 & 0.0052 \\
\hline Triclopyr & 0.0398 & 0.1179 & 0.2211 & 0.1905 & 0.1436 & 0.0520 \\
\hline Trifloxystrobin & 0.3277 & 0.2543 & 3.2789 & 1.2290 & 0.8038 & 0.3767 \\
\hline Vinclozolin & 0.8230 & 0.9713 & 2.9116 & 1.5535 & 1.1562 & 0.7111 \\
\hline
\end{tabular}


Table 3 Dietary pesticide exposure estimates for specific population subgroups (ng/kg/day) ${ }^{a}$ (Continued)

\begin{tabular}{|c|c|c|c|c|c|c|c|c|}
\hline M 14-16 & F 25-30 & M 25-30 & F 40-45 & M 40-45 & F 60-65 & M 60-65 & F 70 & $M 70$ \\
\hline 16.6332 & 16.2023 & 15.7001 & 21.6464 & 16.1272 & 18.6599 & 17.3764 & 20.7385 & 24.0702 \\
\hline 0.0047 & 0.0051 & 0.0053 & 0.0066 & 0.0067 & 0.0068 & 0.0085 & 0.0117 & 0.0138 \\
\hline 8.3018 & 6.9344 & 7.9611 & 9.1004 & 7.1628 & 6.4063 & 10.6951 & 10.3453 & 12.4859 \\
\hline 0.9070 & 1.0642 & 0.3536 & 1.3359 & 0.6003 & 1.4125 & 1.0705 & 1.1712 & 1.1343 \\
\hline 5.7494 & 8.0872 & 5.3303 & 7.9251 & 5.6981 & 15.7172 & 8.8867 & 11.2131 & 9.4220 \\
\hline 1.0640 & 0.8047 & 0.8643 & 0.7484 & 0.6973 & 1.7146 & 1.0494 & 1.3084 & 0.9522 \\
\hline 0.4603 & 0.2670 & 0.2977 & 0.3111 & 0.3271 & 0.2688 & 0.3726 & 0.4180 & 0.4281 \\
\hline 5.6579 & 7.1469 & 4.0346 & 9.5369 & 5.7625 & 7.9005 & 8.7611 & 9.7644 & 9.2733 \\
\hline 10.4841 & 8.4267 & 6.1624 & 7.8695 & 7.7365 & 9.6286 & 8.2916 & 10.5855 & 8.9616 \\
\hline 0.6874 & 0.2739 & 0.0836 & 0.3614 & 0.2877 & 0.2016 & 0.2547 & 0.3386 & 0.4815 \\
\hline 0.0870 & 0.0689 & 0.0728 & 0.0768 & 0.0817 & 0.1271 & 0.0962 & 0.1116 & 0.1194 \\
\hline 0.0416 & 0.0325 & 0.0372 & 0.0370 & 0.0383 & 0.0589 & 0.0503 & 0.0539 & 0.0607 \\
\hline 0.0125 & 0.0091 & 0.0084 & 0.0095 & 0.0113 & 0.0177 & 0.0094 & 0.0132 & 0.0120 \\
\hline 0.0313 & 0.0256 & 0.0257 & 0.0287 & 0.0306 & 0.0464 & 0.0347 & 0.0418 & 0.0441 \\
\hline 0.0016 & 0.0016 & 0.0015 & 0.0016 & 0.0015 & 0.0042 & 0.0018 & 0.0026 & 0.0026 \\
\hline 525.9805 & 249.3211 & 375.2243 & 191.8389 & 269.2749 & 116.9667 & 224.2651 & 158.1203 & 195.8510 \\
\hline 0.1221 & 0.1160 & 0.0956 & 0.1034 & 0.0905 & 0.3776 & 0.1823 & 0.1505 & 0.1970 \\
\hline 2.3484 & 1.8354 & 1.9704 & 2.3210 & 2.0765 & 4.0656 & 2.2079 & 2.4321 & 2.1384 \\
\hline 19.1060 & 9.3327 & 12.5135 & 10.0469 & 11.2686 & 9.4714 & 10.4018 & 10.5705 & 11.0058 \\
\hline 1.0531 & 0.5116 & 0.6111 & 0.4960 & 0.6298 & 0.6130 & 0.7019 & 1.0500 & 1.0474 \\
\hline 0.0059 & 0.0055 & 0.0048 & 0.0056 & 0.0029 & 0.0589 & 0.0034 & 0.0073 & 0.0102 \\
\hline 0.1485 & 0.1835 & 0.3236 & 0.3696 & 0.2331 & 0.3776 & 0.1847 & 0.1690 & 0.2134 \\
\hline 7.3911 & 5.9054 & 7.3996 & 7.4440 & 5.7677 & 16.1349 & 12.9267 & 13.8612 & 9.2338 \\
\hline 4.4096 & 9.4482 & 3.2211 & 12.4248 & 8.1982 & 13.2693 & 11.2770 & 15.4098 & 12.2943 \\
\hline 0.8322 & 0.5248 & 0.6807 & 0.5106 & 0.5936 & 0.2281 & 0.6328 & 0.6007 & 0.6054 \\
\hline 0.1457 & 0.2279 & 0.1898 & 0.2575 & 0.1982 & 0.2792 & 0.2255 & 0.2900 & 0.2151 \\
\hline 10.0929 & 5.3405 & 5.8316 & 4.8189 & 5.9851 & 5.9422 & 6.5278 & 5.2919 & 5.3867 \\
\hline 9.2297 & 4.6048 & 5.4149 & 4.1668 & 5.2854 & 5.1620 & 5.5193 & 4.4752 & 4.6449 \\
\hline 0.0122 & 0.0092 & 0.0099 & 0.0120 & 0.0098 & 0.0188 & 0.0136 & 0.0129 & 0.0123 \\
\hline 0.2212 & 0.1143 & 0.1368 & 0.1157 & 0.1290 & 0.1260 & 0.1384 & 0.1317 & 0.1387 \\
\hline 0.0166 & 0.0240 & 0.0127 & 0.0230 & 0.0196 & 0.0365 & 0.0280 & 0.0404 & 0.0352 \\
\hline
\end{tabular}


Table 3 Dietary pesticide exposure estimates for specific population subgroups ( $\mathrm{ng} / \mathrm{kg} /$ day) ${ }^{a}$ (Continued)

\begin{tabular}{|c|c|c|c|c|c|c|c|c|}
\hline 0.6132 & 0.5882 & 0.2573 & 0.5015 & 0.5414 & 0.5990 & 0.8284 & 0.6318 & 0.5557 \\
\hline 0.1867 & 0.0914 & 0.1604 & 0.0800 & 0.0989 & 0.1099 & 0.0761 & 0.0407 & 0.0376 \\
\hline 0.1779 & 0.2044 & 0.1988 & 0.2714 & 0.2196 & 0.3073 & 0.2134 & 0.1736 & 0.2062 \\
\hline 1.0057 & 0.4768 & 0.5371 & 0.4497 & 0.6017 & 0.5380 & 0.6243 & 0.8806 & 0.8722 \\
\hline 3.5281 & 2.6613 & 2.5448 & 2.4112 & 2.4526 & 6.1333 & 3.5399 & 2.9938 & 3.5544 \\
\hline 3.4838 & 2.6193 & 2.5101 & 2.3737 & 2.4197 & 5.9964 & 3.4738 & 2.9392 & 3.4830 \\
\hline 0.0235 & 0.0245 & 0.0219 & 0.0210 & 0.0225 & 0.0755 & 0.0320 & 0.0532 & 0.0526 \\
\hline 0.2921 & 0.2439 & 0.2535 & 0.2669 & 0.2621 & 0.4260 & 0.3315 & 0.4221 & 0.3968 \\
\hline 1.2978 & 0.7177 & 0.8500 & 0.7267 & 0.8265 & 1.1578 & 0.8707 & 1.0342 & 0.9787 \\
\hline 1.3173 & 1.3197 & 1.0581 & 1.6276 & 1.3318 & 2.3255 & 1.2750 & 1.3512 & 1.5124 \\
\hline 65.1788 & 48.8023 & 64.6768 & 70.0400 & 50.1053 & 21.8490 & 78.2527 & 64.3824 & 81.6502 \\
\hline 10.0291 & 7.2010 & 8.0732 & 8.6182 & 7.8821 & 11.6984 & 8.2978 & 9.3896 & 9.2824 \\
\hline 1.8862 & 1.2782 & 1.4997 & 1.4701 & 1.4373 & 1.5188 & 1.3303 & 1.3647 & 1.3876 \\
\hline 2.7102 & 1.7453 & 2.0406 & 2.0020 & 1.7985 & 2.3578 & 1.8384 & 1.8595 & 1.8490 \\
\hline 5.4327 & 4.1775 & 4.5329 & 5.1461 & 4.6463 & 7.8219 & 5.1291 & 6.1655 & 6.0459 \\
\hline 0.0108 & 0.0202 & 0.0118 & 0.0198 & 0.0153 & 0.0323 & 0.0191 & 0.0384 & 0.0349 \\
\hline 0.0329 & 0.0152 & 0.0231 & 0.0183 & 0.0161 & 0.1703 & 0.0291 & 0.0398 & 0.0277 \\
\hline 0.9142 & 0.4462 & 0.5556 & 0.4021 & 0.4089 & 0.5313 & 0.2811 & 0.2328 & 0.2838 \\
\hline 0.9111 & 0.4409 & 0.5519 & 0.3982 & 0.4049 & 0.5255 & 0.2792 & 0.2307 & 0.2810 \\
\hline 0.0031 & 0.0053 & 0.0037 & 0.0039 & 0.0040 & 0.0057 & 0.0019 & 0.0020 & 0.0028 \\
\hline 0.7313 & 0.6021 & 0.7855 & 0.6930 & 0.6076 & 2.0313 & 0.3458 & 0.2589 & 0.3347 \\
\hline 0.0793 & 0.0980 & 0.1728 & 0.1973 & 0.1244 & 0.2016 & 0.0986 & 0.0902 & 0.1139 \\
\hline 0.0032 & 0.0145 & 0.0184 & 0.0167 & 0.0156 & 0.0521 & 0.0155 & 0.0212 & 0.0312 \\
\hline 0.6234 & 0.8525 & 0.2541 & 1.0779 & 0.5421 & 1.1354 & 0.8992 & 1.0622 & 0.9554 \\
\hline 0.0091 & 0.0111 & 0.0110 & 0.0176 & 0.0114 & 0.0307 & 0.0082 & 0.0065 & 0.0057 \\
\hline 0.0000 & 0.0000 & 0.0000 & 0.0000 & 0.0000 & 0.0130 & 0.0000 & 0.0000 & 0.0004 \\
\hline 2.0885 & 2.1623 & 2.1035 & 3.0221 & 2.2667 & 3.5484 & 3.2033 & 3.4172 & 3.5806 \\
\hline 0.2464 & 0.2754 & 0.2897 & 0.3427 & 0.3419 & 0.6703 & 0.4263 & 0.6357 & 0.7162 \\
\hline 13.5739 & 18.9030 & 7.6395 & 24.7306 & 19.2154 & 18.2427 & 20.6759 & 27.8568 & 17.7320 \\
\hline 0.0816 & 0.0474 & 0.0569 & 0.0443 & 0.0543 & 0.0740 & 0.0428 & 0.0483 & 0.0468 \\
\hline 0.4188 & 0.3255 & 0.2403 & 0.5650 & 0.4071 & 0.6250 & 0.7987 & 1.5949 & 1.7592 \\
\hline 3.7946 & 5.6403 & 2.2778 & 7.4015 & 5.6484 & 6.2786 & 6.2383 & 8.3525 & 5.5698 \\
\hline 3.5852 & 5.3320 & 2.1398 & 6.9979 & 5.3389 & 5.9016 & 5.8986 & 7.9255 & 5.2641 \\
\hline
\end{tabular}


Table 3 Dietary pesticide exposure estimates for specific population subgroups ( $\mathrm{ng} / \mathrm{kg} /$ day) ${ }^{\text {a }}$ (Continued)

\begin{tabular}{|c|c|c|c|c|c|c|c|c|}
\hline 0.2094 & 0.3083 & 0.1380 & 0.4036 & 0.3095 & 0.3771 & 0.3397 & 0.4270 & 0.3057 \\
\hline 0.2008 & 0.4422 & 0.4518 & 0.8299 & 0.4960 & 1.7703 & 0.9220 & 0.8178 & 0.7633 \\
\hline 0.1176 & 0.0677 & 0.0548 & 0.0509 & 0.0754 & 0.1302 & 0.0425 & 0.0358 & 0.0397 \\
\hline 0.1302 & 0.2156 & 0.1544 & 0.2746 & 0.2104 & 0.3906 & 0.2221 & 0.1815 & 0.2134 \\
\hline 42.7260 & 21.6705 & 29.3563 & 22.6826 & 26.6826 & 18.7036 & 25.9992 & 24.2481 & 25.5719 \\
\hline 0.3646 & 0.7334 & 0.4357 & 1.0752 & 0.7592 & 1.1266 & 0.9553 & 1.2109 & 1.0349 \\
\hline 6.0969 & 6.0924 & 7.5724 & 8.8198 & 7.6102 & 8.5927 & 8.3945 & 9.4966 & 10.7210 \\
\hline 1.5486 & 0.7018 & 0.9954 & 0.6535 & 0.6442 & 0.2578 & 0.7235 & 0.9699 & 0.7849 \\
\hline 1.5201 & 1.6876 & 1.0198 & 2.2454 & 1.3298 & 1.9276 & 1.7928 & 1.9009 & 1.8352 \\
\hline 0.0331 & 0.0443 & 0.0364 & 0.0434 & 0.0402 & 0.0474 & 0.0454 & 0.0268 & 0.0340 \\
\hline 0.0005 & 0.0215 & 0.0150 & 0.0220 & 0.0194 & 0.0042 & 0.0214 & 0.0118 & 0.0162 \\
\hline 0.0326 & 0.0228 & 0.0214 & 0.0214 & 0.0208 & 0.0432 & 0.0241 & 0.0150 & 0.0178 \\
\hline 0.0012 & 0.0017 & 0.0014 & 0.0016 & 0.0016 & 0.0021 & 0.0022 & 0.0021 & 0.0019 \\
\hline 0.6311 & 0.7045 & 0.7028 & 0.9551 & 0.7554 & 1.4615 & 0.8879 & 0.8878 & 0.8731 \\
\hline 0.0387 & 0.0272 & 0.0453 & 0.0432 & 0.0400 & 0.0714 & 0.0536 & 0.0423 & 0.0630 \\
\hline 80.2934 & 57.2709 & 66.1862 & 56.3328 & 48.4414 & 130.2292 & 82.2046 & 101.4862 & 72.1103 \\
\hline 38.5280 & 27.6290 & 31.8599 & 27.3468 & 23.4409 & 62.8005 & 39.6630 & 48.7888 & 34.8039 \\
\hline 41.7654 & 29.6419 & 34.3263 & 28.9859 & 25.0005 & 67.4286 & 42.5416 & 52.6974 & 37.3064 \\
\hline 23.6654 & 7.2615 & 6.2418 & 10.2150 & 9.1555 & 27.4469 & 13.1577 & 17.8501 & 14.0892 \\
\hline 0.0464 & 0.0734 & 0.0799 & 0.0449 & 0.0500 & 0.0703 & 0.0111 & 0.0071 & 0.0156 \\
\hline 3.2591 & 3.8788 & 2.2576 & 5.1557 & 3.9565 & 4.5583 & 4.6251 & 5.6095 & 4.2228 \\
\hline 0.0000 & 0.0000 & 0.0000 & 0.0000 & 0.0000 & 0.1823 & 0.0000 & 0.0000 & 0.0000 \\
\hline 8.1045 & 5.2557 & 6.2427 & 5.4557 & 4.8230 & 8.8276 & 5.5365 & 3.4975 & 3.7984 \\
\hline 0.0648 & 0.0205 & 0.0188 & 0.0278 & 0.0245 & 0.0266 & 0.0167 & 0.0188 & 0.0194 \\
\hline 6.6315 & 9.0565 & 5.8634 & 12.0346 & 9.6961 & 19.5833 & 10.0678 & 15.6229 & 13.0497 \\
\hline 0.0000 & 0.0000 & 0.0000 & 0.0000 & 0.0000 & 0.0130 & 0.0000 & 0.0000 & 0.0004 \\
\hline 1.2727 & 1.1870 & 1.1458 & 0.9132 & 0.9463 & 0.4484 & 0.7726 & 0.5402 & 0.4990 \\
\hline 0.0542 & 0.1524 & 0.0542 & 0.2101 & 0.1428 & 0.2083 & 0.1836 & 0.2548 & 0.1979 \\
\hline 0.0740 & 0.0637 & 0.0553 & 0.0663 & 0.0544 & 0.0693 & 0.0682 & 0.0857 & 0.0783 \\
\hline 0.0013 & 0.0006 & 0.0009 & 0.0007 & 0.0008 & 0.0005 & 0.0008 & 0.0008 & 0.0009 \\
\hline 70.3642 & 49.8231 & 61.0063 & 63.9146 & 52.3844 & 42.6089 & 75.2598 & 78.1656 & 84.9818 \\
\hline 0.0013 & 0.0032 & 0.0041 & 0.0019 & 0.0024 & 0.0052 & 0.0017 & 0.0018 & 0.0020 \\
\hline 0.0901 & 0.0397 & 0.0396 & 0.0265 & 0.0275 & 0.0339 & 0.0194 & 0.0150 & 0.0138 \\
\hline 0.0813 & 0.2287 & 0.0813 & 0.3152 & 0.2141 & 0.3125 & 0.2754 & 0.3822 & 0.2968 \\
\hline 0.5821 & 0.7231 & 0.3974 & 0.8727 & 0.5666 & 0.9401 & 0.8155 & 0.9626 & 0.9968 \\
\hline
\end{tabular}

a Levels in bold represent highest exposed population subgroup 
malathion (29.7 ng/kg/day), acephate (18.4 ng/kg/day), chlorpyrifos-methyl $(12.8 \mathrm{ng} / \mathrm{kg} /$ day $)$, and azinphosmethyl $(11.1 \mathrm{ng} / \mathrm{kg} /$ day). The reason that methamidophos poses a much greater exposure relative to the chronic RfD than do the other organophosphate insecticides is that it has an extremely low chronic RfD of $50 \mathrm{ng} / \mathrm{kg} /$ day. This low RfD is derived partly from the use of a 1000 fold uncertainty factor (rather than the typical 100 fold) and also from a toxicologically suspect low NOAEL of $50 \mu \mathrm{g} / \mathrm{kg} /$ day. Comparisons of the LD50 values for methamidophos and some of the most acutely toxic organophosphate insecticides do not indicate such disparities in toxicity.

The only other pesticides showing dietary exposure greater than $1 \%$ of the chronic RfD were dieldrin $(2.0 \%$ of chronic RfD) and DDT (1.3\% of chronic RfD). Both of these pesticides are chlorinated hydrocarbon insecticides that have been banned from use for decades; their presence in food results from low environmental degradation and uptake from contaminated soil by plants.

Several other chlorinated hydrocarbon insecticides were also detected in the 2004-2005 TDS. To indicate trends in exposure to chlorinated hydrocarbon insecticides over time, it is possible to compare these findings with those of prior years. Table 2 shows the reduction in dietary exposure levels for two year-olds (the population subgroup associated with the greatest dietary exposure to pesticides) from dietary estimates using the 1986-1991 TDS data (Gunderson 1995) to dietary estimates using the 2004-2005 TDS data. Dietary exposures were reduced by between 47 and $96 \%$ from 1986-1991 to 2004-2005 for the six chlorinated hydrocarbon insecticides studied.

Chronic dietary exposure estimates to the 77 pesticides for each of the 15 population subgroups is shown in Table 3. While there is significant variation in the exposure estimates for specific pesticides among the population subgroups, all exposures to all population subgroups were below chronic RfD levels. Table 3 also indicates that the highest exposures are typically observed in the younger population subgroups, as is expected since younger members of the population consume more food relative to their body weights than do older members of the population. For 51 of the 77 pesticides, population subgroup exposure was highest for two year-olds, while another 13 pesticides showed highest exposure for 6-11 month infants. Exposure to six year-olds was highest for 6 pesticides and exposure to 60-65 year-old females was highest for 4 pesticides. Three other population subgroups (10 year-olds, 14-16 year-old males, 70 year-old males) showed the highest dietary exposure to one pesticide each.

This paper provides estimates of chronic exposure to pesticides based upon mean residue levels found in the TDS and reliance on average food consumption patterns for the General US population and for population subgroups. As such, efforts were not made to assess acute toxicity risks from pesticide residue exposure to determine the likelihood that a single person's dietary exposure to a pesticide could exceed the acute RfD on a given day. More sophisticated risk assessment methods, using probabilistic models to study variability in pesticide residue levels and food consumption data, would be needed to achieve this task. Such approaches are commonly performed by the EPA, however, and pesticides are not allowed to be registered for use unless EPA concludes that the pesticides pose a "reasonable certainty of no harm" when considering potential increased susceptibility for specific population subgroups, aggregate exposure to pesticides (water, food, and residential exposure) and cumulative exposure to families of pesticides possessing common mechanisms of toxicological action such as the organophosphate insecticides. For acute pesticide exposure, a pesticide poses a "reasonable certainty of no harm" when it can be established that acute exposure for sensitive population subgroups has at least a $99.9 \%$ chance of being below the acute RfD (EPA 2014).

\section{Conclusions}

The findings from analysis of TDS pesticide residue data confirm that while pesticide residues are frequently detected in a variety of food products, chronic dietary exposure to pesticides continues to be at levels far lower than levels considered to be of health concern. Consumer fears from pesticide residues provide the potential for consumers to reduce their consumption of fruits, vegetables, and grains, negating the positive health benefits attributed to consumption of large amounts of such foods in one's diet. Findings from this study also indicate that the potential health benefits from further reducing one's exposure to pesticide residues through purchase of organic foods may not provide any appreciable benefit given the very low level of pesticide residues consumers are typically exposed to from conventionally produced foods and the finding that organic foods commonly have been shown to contain pesticide residues as well, although at lower frequency than their conventional counterparts (Winter 2012).

While the findings from this paper represent the most recently released TDS data for pesticide residues, such findings are still at least ten years old and may not reflect pesticide residue levels that are encountered today. Food consumption data is similarly dated, representing results from dietary surveys conducted in 1994-96 and in 1998. Fortunately, the methodology to develop exposure estimates for pesticides can be easily adapted to more contemporary residue and food consumption data when such data become available. At the present time, however, the methodology used in this paper provides a convenient and comprehensive approach to estimate 
chronic dietary exposure to pesticides and to compare such exposures with chronic RfD levels. Unless significant changes in pesticide use patterns and food consumption behavior become evident, it is unlikely that the use of more contemporary data will alter the conclusion that chronic dietary exposure to pesticides is typically well below chronic RfD levels.

\section{Competing interests}

The author declares that he has no competing interests.

Received: 17 April 2015 Accepted: 27 June 2015

Published online: 10 July 2015

\section{References}

EPA Environmental Protection Agency (2011) Exposure factors handbook. http://www.epa.gov/ncea/efh/pdfs/efh-complete.pdf. Accessed 15 Apr 2015

EPA Environmental Protection Agency (2014) The Food Quality Protection Act (FQPA) background. http://www.epa.gov/pesticides/regulating/laws/fapa/ backgrnd.htm. Accessed 15 Apr 2015

EFSA European Food Safety Authority (2015) The 2013 European Union report on pesticide residues in food. http://www.efsa.europa.eu/en/efsajournal/pub/ 4038.htm. Accessed 15 Apr 2015

FDA Food and Drug Administration (2013) Total diet study diets, version 3. http://www.fda.gov/Food/FoodScienceResearch/TotalDietStudy/ ucm184232.htm\#fca. Accessed 15 Apr 2015

FDA Food and Drug Administration (2014) Total diet study market baskets 2004-1 through 2005-4. http://www.fda.gov/downloads/Food/FoodScienceResearch/ TotalDietStudy/UCM291686.pdf Accessed 15 Apr 2015

FDA Food and Drug Administration (2015) Pesticide monitoring program fiscal year 2012 pesticide report. http://www.fda.gov/downloads/Food/Foodbor nelllnessContaminants/Pesticides/UCM432758.pdf. Accessed 15 Apr 2015

Gunderson EL (1995) FDA Total Diet Study, July 1986-April 1991, dietary intakes of pesticides, selected elements, and other chemicals. J AOAC Int 78(6):1353-1363

Winter CK (1992) Pesticide tolerances and their relevance to safety standards. Regul Toxicol Pharmacol 15:137-150

Winter CK (2012) Pesticide residues in imported, organic, and "suspect" fruits and vegetables. J Agric Food Chem 60:4425-4429

Winter CK, Francis FJ (1997) Assessing, managing, and communicating food safety risks. Food Technol 51(5):85-92

Winter CK, Jara EA (2015) Pesticide food safety standards as companions to tolerances and maximum residue limits. J Integr Agric (in press).

\section{Submit your manuscript to a SpringerOpen ${ }^{\circ}$} journal and benefit from:

- Convenient online submission

- Rigorous peer review

- Immediate publication on acceptance

- Open access: articles freely available online

- High visibility within the field

- Retaining the copyright to your article 